
8

\title{
Serum fibroblast growth factor 19 as a predictor for early stage of gastrointestinal-liver dysfunction in pediatric patients with sepsis
}

\section{Xiaomeng Tang}

Shanghai Children's Hospital

\section{Yuqian Ren}

Shanghai Children's Hospital

Chunxia Wang ( $\sim$ karencx0465@163.com )

Shanghai Children's Hospital https://orcid.org/0000-0003-4550-5208

\section{Yun Cui}

Shanghai Children's Hospital

\section{Xi Xiong}

Shanghai Children's Hospital

\section{Huijie Miao}

Shanghai Children's Hospital

\section{Ting Sun}

Shanghai Children's Hospital

Ye Lu

Shanghai Children's Hospital

\section{Lujing Shao}

Shanghai Children's Hospital

\section{Yucai Zhang}

Shanghai Children's Hospital

\section{Research article}

Keywords: FGF19, gastrointestinal dysfunction, liver injury, sepsis

Posted Date: August 30th, 2019

DOl: https://doi.org/10.21203/rs.2.11560/v1

License: (a) (i) This work is licensed under a Creative Commons Attribution 4.0 International License.

Read Full License 


\section{Abstract}

Background: Fibroblast growth factor 19 (FGF19) secrets from intestinal epithelial cells and proved to be associated with functioning ileum in Crohn's disease and alcoholic steatohepatitis. In the present study, we aimed to explore the potential value of FGF19 as a biomarker for assessment of gastrointestinal (GI) dysfunction in pediatric patients with sepsis.

Methods: We performed a prospective study to enroll pediatric patients with sepsis admitted to the pediatric intensive care unit (PICU) at Shanghai Children's Hospital from January 2018 to December 2018. Serum FGF19 levels at PICU admission were determined, and the clinical and laboratory parameters were collected.

Results: Significantly decreased serum FGF19 levels were found in patients with sepsis-associated GI dysfunction. Lower serum FGF19 levels than $60 \mu \mathrm{g} / \mathrm{mL}$ on PICU admission is predictive for GI dysfunction. Correlation analyses revealed significant correlations of serum FGF19 with hemoglobin, and low FGF19 serum concentration less than $52 \mu \mathrm{g} / \mathrm{mL}$ on PICU admission is related to GI dysfunctionassociated liver injury.

Conclusion: Serum FGF19 is a novel predictor for GI dysfunction or Gl-liver dysfunction in pediatric patients with sepsis.

\section{Introduction}

Acute gastrointestinal injury (AGI) is common in intensive care unit and associated with worse prognosis in critically ill patients [1]. The Gastrointestinal (GI) plays a crucial role in the pathophysiology of sepsis [2]. Conversely, sepsis is potential concerns with $A G I$, and resuscitation from septic shock may be associated with morphologic changes in GI epithelium resulting in GI disorder, GI motor dysfunction, GI barrier dysfunction, etc. [3]. Consequently, GI barrier dysfunction provides an outlet for intestinal flora moving to other locations, leading to the aggravation of sepsis with multiple organ dysfunction syndrome (MODS) [4]. Specially, intestinal failure was closely related to occurrence of liver disorder [5]. Considering of the hypothesis of $\mathrm{Gl}$ as the motor of sepsis, early identification of AGI and stable intestinal barrier are critical for against the progress of sepsis.

The signs and symptoms about AGI include abnormal bowel sounds, abdominal distention and pain, diarrhea, constipation, nausea, vomiting, inability to tolerate enteral feeding, and GI hemorrhage. The grade of AGI based on European Society of Intensive Care Medicine (ESICM) strongly affected by the subjective factors [6]. Up to date, easily applicable variable(s) and biomarkers for AGI are not available. So, it is necessary to develop the sensitive and specific biomarker to early or timely diagnose AGI. The mucosal barrier of GI mostly consists of epithelial cells. Intestinal epithelial cells-drived "signaling message" might contribute to the intestinal-other organs crosstalk. Fibroblast growth factor 19 (FGF19) secrets from intestinal epithelial cells and involves in the feedback regulation of bile acid synthesis mediating the communication between the small intestine and the liver [7]. Limited studies indicated that 
reduced FGF19 levels are associated with ileal resection, diarrhea and disease activity, and FGF19 may as a biomarker for functioning ileum in Crohn's disease [8, 9]. Adiponectin-FGF15/19 axis as an essential adipose-GI-liver coordinator involved in the development and progression of alcoholic steatohepatitis [10]. Furthermore, administration of a nontumorigenic FGF19 variant (M70) in healthy human volunteers represents an effective approach for the prevention and treatment of cholestatic liver diseases associated with bile acid dysregulation via potently reducing the hepatic activity of cholesterol 7a-hydroxylase (CYP7A1) [11]. All these reports suggest that FGF19 levels might correlated with the Gl-liver function.

It is unknown whether serum FGF19 plays a key role in patients with sepsis as a "message" or "biomarker" for Gl-liver dysfunction. So, we performed a prospective observational study and collected serum from pediatric patients with sepsis to investigate the potential role of FGF19 as a diagnostic biomarker for or Gl-liver dysfunction.

\section{Materials And Methods}

\subsection{Study Design and Patient Characteristics}

Patients with sepsis were enrolled in this study from January 2018 to December 2018. Sepsis were diagnosed based on the International Pediatric Sepsis consensus conference in 2005 [12] and Surviving Sepsis Campaign International Guidelines in 2012 [13]. Only patients who were expected to have a longterm (> 72hr) intensive care treatment were included into this study. The patients with advanced tumor or life expectancy less than 1 month were excluded. Patients diagnosed with sepsis over $24 \mathrm{~h}$ were excluded. Sepsis-associated GI dysfunction was defined according to the guideline by European Society of Intensive Care Medicine (ESICM) [6]. Sepsis-associated acute kidney injury (AKI) was defined as infectious episode with AKI according to the KDIGO criteria [14]. Sepsis-associated liverinjury (SALI) were diagnosed according to the criteria of International pediatric sepsis consensus conference [12]. SALI was defined by the following conditions: 1) TBIL $\geq 4.0 \mathrm{mg} / \mathrm{dL}$, or 2) ALT 2 times upper limit of normal for age. The study protocol was approved by the local ethics committee and conducted in accordance with the ethical standards laid down in the Declaration of Helsinki (Ethics Committee of Children's Hospital affiliated to Shanghai Jiao Tong university (Approval number: 2018R039-F01). The informed consent was signed by the patients' relatives.

\subsection{Patient's treatment}

Patients were treated with fluid therapy, vasoactive drugs, antibiotics, and other supportive therapy recommended by the International Pediatric Sepsis consensus conference in 2005 [12] and Surviving Sepsis Campaign International Guidelines in 2012 [13].

\subsection{Blood samples}


Blood samples were collected without anticoagulant at the time point of patient's admission to the PICU. After centrifugation, the serum was stored at $-80^{\circ} \mathrm{C}$. Serum FGF19 levels were determined using Enzymelinked immunosorbent assays (ELISA) (MultiScience [LIANKE] Biotech, CO., LTD, Hangzhou, China).

\subsection{Observational variables}

According to the pre-established Case Report Form (CRF), we collected the clinical parameters including age, sex, co-morbidities, infection sources, PRISM III score, mechanical ventilator, vasoactive agents. The laboratory indexes include routine blood indexes (platelet, PLT; the percentage of natural killer [NK], T cells $\left[\mathrm{CD} 4^{+} \mathrm{CD}^{+}\right]$; and $\mathrm{B}$ cells $\left[\mathrm{CD} 19^{+}\right]$) and biochemical parameters for organ functions (total bilirubin, TBIL; alanine aminotransferase, ALT; albumin; blood urea nitrogen, BUN; creatinine, $\mathrm{Cr}$; lactic acid, Lac; creatine kinase isoenzyme MB, CK-MB), coagulation function (activated partial thromboplastin time, APTT; international normalized ratio, INR; fibrinogen, Fib), and infectious indexes (procalcitonin, PCT; c-reaction protein, CRP; white blood cell, WBC). The outcome variables included the length of PICU stay and PICU survival status. The laboratory indexes were collected from the first test within 24 hours after PICU admission.

\subsection{Statistical Analyses}

Continuous variables were presented as median (Inter Quartile Range, IQR) for abnormal distribution data and as means \pm standard derivations (SD) for normal distribution data. Student $t$ test and the MannWhitney $\mathrm{U}$ test were used to compare the continuous variables with normally distributed data or abnormal distribution, repectively. The chi-square test was used to compare the categorical data. Unadjusted associations between covariates and GI dysfunction were estimated by bivariable logistic regression models. Adjusted odd ratios (ORs) were estimated by multivariate logistic regression models with inclusion of covariate terms chosen based on the biological plausibility of possible confounding of laboratory indexes and outcome. Correlation analysis was performed based on linear regression. In order to appreciate the accuracy of independently predictors of sepsis-associated GI dysfunction, a ROC curve was generated. Data analyses were performed using STATA 15.0 MP (College Station, Texas, USA). A value of $P<0.05$ was considered statistically significant.

\section{Results}

\subsection{Baseline characteristics of patients}

A total of 101 patients were enrolled, and 11 cases without FGF19 value were excluded. Finally, there were 90 cases (51 males and 49 females) used to further analysis with a median age of $19(5,60)$ months.

The baseline characteristics of patients enrolled in this study were shown in Table 1. The PICU morbidity rate of pediatric sepsis was $12.2 \%$ (11/90). According to GI dysfunction, the patients were divided into the 
sepsis group $(n=58)$ and the sepsis-associated GI dysfunction group $(n=32)$. There were significant differences between two groups in aspects of PRISM III score, rate of shock, AKI, or acute liver injury (ALI), infection site, and the length of PICU stay (all $P<0.05$ ). In addition, PICU mortality in the group of GI dysfunction showed a higher tendency but without statistic significance compared with that in the non-GI dysfunction group $(18.8 \%$ vs. $8.6 \%, P=0.160)$ Table 1$)$..

\subsection{Serum FGF19 levels are specifically correlated with GI dysfunction in pediatric patients}

Based on our initial hypothesis, we further investigated if the occurrence of GI dysfunction might have a specific impact on circulating FGF19. Remarkably, we found significantly decreased FGF19 serum concentrations at PICU admission in patients who fulfilled septic GI dysfunction criteria, compared with sepsis without $\mathrm{Gl}$ dysfunction $(48.4[27.7,95.6] \mu \mathrm{g} / \mathrm{ml} v s .77 .6[45.8,151.2] \mu \mathrm{g} / \mathrm{ml}, P=0.046$, Table 2).. Moreover, the levels of PCT were significantly higher $(4.5[0.6,12.6] \mathrm{ng} / \mathrm{mL}$ vs. $0.5[0.1,5.7] \mathrm{ng} / \mathrm{mL}, P=$ 0.005), but the levels of albumin were significantly lower in patients with $\mathrm{Gl}$ dysfunction than patients without $\mathrm{Gl}$ dysfunction $(36.4[28.3,40.3] \mathrm{g} / \mathrm{L}$ vs. $39.6[34.5,43.1] \mathrm{g} / \mathrm{L}, P=0.025$, Table 2$)$.. In addition, serum lactate levels and INR values displayed higher tendency but without statistic significance $(P=$ $0.126, P=0.116$, respectively; Table 2 )

To investigate the influence of sepsis-associated organ dysfunction precisely, we performed subgroup analysis among patients with sepsis. When patients were further subdivided into subgroups with different organ dysfunction, the levels of FGF19 were no difference with the occurrence of sepsisassociated respiratory failure $(P=0.731), \operatorname{AKI}(P=0.373)$, or acute $\operatorname{ALI}(P=0.426)$, septic shock $(P=$ 0.053). Furthermore, FGF19 serum concentrations did not correlate with age or sex in patients with sepsis $(P=0.925, P=0.495$; respectively).

Additionally, multivariate logistic regression analysis indicated that low FGF19 level was independently risk factor for the occurrence of GI dysfunction in pediatric patients with sepsis (OR: 0.992, 95\% confidence interval [CI]:: 0.984-0.999, $P=0.046$ ) adjusted by PCT, albumin, Lac, INR, and PRISM III score (Table 3)..

\subsection{Serum FGF19 concentration is a predictive marker for GI dysfunction in pediatric patients with sepsis}

When we applied ROC analyses to evaluate the diagnostic value of FGF19 levels in pediatric patients with sepsis, FGF19 serum levels achieved similar area under the ROC (AUC) statistics as PCT (AUC: 0.636 $[0.515,0.757]$ vs. 0.683 [0.562, 0.804], $P=0.597$; Figure. 1).. Furthermore, FGF19 serum levels displayed the diagnostic accuracy for GI dysfunction with a sensitivity of $62.5 \%$ and a specificity of $55.2 \%$ with the cutoff value of $60 \mu \mathrm{g} / \mathrm{mL}$. Though PCT was no independent risk factor for diagnosis of GI dysfunction, 


\subsection{Levels of circulating FGF19 are closely correlated to serum albumin levels and liver injury}

Given the critical role of FGF19 in cross-talk between GI and liver, we tried to evaluate the correlation of FGF19 levels and liver injury in pediatric patients with sepsis. The serum FGF19 levels were positively correlated with the serum albumin levels at the time of PICU admission $\left(r^{2}=0.056, P=0.024 ;\right.$ Figure 2A).. In addition, though there was no significant difference in total patients with or without liver injury (Figure 2B),, serum FGF19 levels were significantly lower in subgroup of GI dysfunction complicated with ALI than that without ALI (Figure 2C).. Though serum FGF19 showed a poorly diagnostic potential in sepsisassociated liver injury in the present small size, the rate of liver injury had a higher tendency in the group of patients with FGF19 $<52 \mu \mathrm{g} / \mathrm{mL}$, which were obtained by ROC analysis as a threshold value for the occurrence of liver injury (Figure 2D)..

\section{Discussion}

In the present study, we found that serum FGF19 levels are correlated with the occurrence of GI dysfunction, which also is a potential biomarker for GI dysfunction-associated liver injury with the threshold value of $52 \mu \mathrm{g} / \mathrm{mL}$.

Pediatric critically ill patients often have a constellation of symptoms due to intra-abdominal hypertension. Several studies indicated that there are up to $62 \%$ of patients exhibiting at least one $\mathrm{GI}$ symptom for at least 1 day after ICU admission $[1,15,16]$. In the present study, the incidence of GI dysfunction in pediatric patients with sepsis was 35.6\% (32/90). Accumulated evidence confirmed that that development of GI problems is related to worse outcome in critically ill patients [15, 17-19]. In adult critically ill patients, the presence of three or more of GI dysfuntion symptoms on the first day in an ICU stay is associated with a three-fold increase in mortality of patients needing mechanical ventilation (MV) [20]. Consistently, the mortality was $6 / 32$ in patients with GI dysfunction with higher tendency mortality than that in children without GI dysfunction (5/58) in our present study.

The lack of markers for the measurement of GI function has suppressed studies in the field of GI dysfunction. Recent report proved that PCT level could distinguish upper or lower GI perforation (GIP) [21]. Given that serum PCT concentration is related to bacterial load [22], the severity of sepsis [23], and even prognosis [24, 25], serum PCT levels might related to late phase of infection. So far, the evidence regarding the diagnostic validity and accuracy of PCT in predicting GI dysfunction in sepsis is lacking. In our present study, though serum PCT concentration was not an independent factor for GI dysfunction, it displayed the AUC of 0.683 for identification of GI dysfunction with a sensitivity of $67.7 \%$ and a specificity of $70.9 \%$ with the cutoff value of $1.44 \mathrm{ng} / \mathrm{mL}$. To the best of our knowledge, this study is the 
first to evaluate the role of PCT in predicting GI dysfunction in sepsis as a useful supplementary tool. Considering that PCT is a regular clinical parameter, PCT would be helpful biomarker for assessing the GI dysfunction in patients with sepsis.

The enterocyte damage occurring in early stage of sepsis affects the secretion of enterocyte-derived factors. These biofactors may help the intensivist to identify GI dysfunction in the early stage. Recently, intestinal fatty acid binding protein (I-FABP) in urine has been proposed as possible markers for the damage of intestinal epithelial cells [26]. In our present study, FGF19, as an enterocyte-derived factor, is significantly correlated with GI dysfunction in patients with sepsis, which gave a new insight into assessment of GI dysfunction. Furthermore, serum FGF19 has a certain power to identify GI dysfunction with a sensitivity of $62.5 \%$ and a specificity of $55.2 \%$ with the cutoff value of $60 \mu \mathrm{g} / \mathrm{mL}$, although the accuracy of FGF19 is moderate. The feasibility of serum FGF19 as a common indicator of GI dysfunction still needs to be confirmed in large size samples. Moreover, FGF19 is involved in the feedback regulation of bile acid synthesis via potently reducing the hepatic activity of CYP7A1 [11], which plays a critical role in the communication between the small intestine and the liver [7]. FGF19 was proved to be correlated with the development and progression of alcoholic steatohepatitis [10]. Interestingly, we found that the lower serum FGF19 level was correlated with GI dysfunction complicated by ALI, and lower FGF19 than $52 \mu \mathrm{g} / \mathrm{ml}$ suggested a higher risk of occurrence of ALI. The difference was not statistic significance possibly due to a small simple size. To our best knowledge, it is the first biomarker displaying the potential value in diagnosis of Gl-liver dysfunction in sepsis. This needs to be paid attention and confirms in a larger sample size of patients with sepsis.

There were several limitations in our study. We prospectively enrolled the patients with sepsis. However, the sample size of sepsis-associated GI dysfunction was relative small. In addition, we did not determine I-FABP as a reference biomarker in the enrolled patients. Furthermore, it would be necessary to analyze the pathology or pathogen of GI-liver injury in patients with sepsis to accurately define the value of FGF19 involved in sepsis-associated GI dysfunction and liver injury.

In summary, serum FGF19 could serve as a new biomarker in pediatric patients with sepsis to assess GI dysfunction and GI dysfunction-associated liver injury. Serum FGF19 concentration less than $60 \mu \mathrm{g} / \mathrm{mL}$ on PICU admission is predictive for GI dysfunction, and less than $52 \mu \mathrm{g} / \mathrm{mL}$ on PICU admission is related to GI dysfunction-associated liver injury.

\section{Abbreviations}

ALT: Alanine aminotransferase;

AUC:Area under the ROC curve

APTT: Activated partial thromboplastin time;

BUN: Blood urea nitrogen; 
CK-MB: Creatine kinase isoenzyme MB;

CRP: C-reactive protein;

Cr: Creatinine;

ELISA:Enzyme-linked immunosorbent assays

FGF19:Fibroblast growth factor 19;

Fib: Fibrinogen;

Gl:Gastrointestinal

Lac: Lactate;

NK: Natural killer.

PCT: Procalcitonin;

PICU:Pediatric intensive care unit

PLT:Platelet;

PRISM $₫$ : Pediatric risk of mortality $₫$ score;

INR:International normalized ratio

ROC:Receiver operating characteristic

TBIL:Total bilirubin;

WBC: White blood cell;

\section{Declarations}

Ethics approval and consent to participate: This study was approved by Ethical Committee of Children's Hospital Affiliated to Shanghai Jiao Tong University and conducted in accordance with provisions of the Declaration of Helsinki (Approval number: 2018R039-F01). The written informed consents were obtained from all patients' parents.

Consent for publication: Not applicable.

Availability of data and materials: Our present study was a retrospective observational study. All the data were obtained from medical records of patients. The raw data was submitted as a supplementary data.

Competing interests: The authors have declared that no competing interests exist. 
Funding: This study was supported by the Shanghai Municipal Education Commission-Gaofeng Clinical Medicine Grant Support (20171928), the Shanghai Municipal Commission of Health and Family Planning (201740025), the Science and Technology Commission of Shanghai Municipality (18411951000, 16411970300), the Talent program from Shanghai Jiao Tong University School of Medicine (17XJ11018), and Natural Science Foundation of Shanghai (19ZR1442500).

Authors' Contributions: Conceived and designed the study: Chunxia Wang and Yucai Zhang, Collected and analyzed data: Xiaomeng Tang, Yuqian Ren, Chunxia Wang, Xi Xiong, Huijie Miao, Ting Sun and Ye Lu, Contributed analysis tools and discussion: Xiaomeng Tang and Chunxia Wang, Wrote the paper: Xiaomeng Tang, Chunxia Wang and Yucai Zhang.

Acknowledgements: We thank for the support from Shanghai Municipal Education Commission-Gaofeng Clinical Medicine Grant Support (20171928), the Shanghai Municipal Commission of Health and Family Planning (201740025), the Science and Technology Commission of Shanghai Municipality $(18411951000,16411970300)$, the Talent program from Shanghai Jiao Tong University School of Medicine (17XJ11018), and Natural Science Foundation of Shanghai (19ZR1442500).

\section{Reference}

1.Reintam A, Parm P, Kitus R, Kern H, Starkopf J: Gastrointestinal symptoms in intensive care patients. Acta Anaesthesiol Scand 2009, 53(3):318-324.

2. Yu Y, Yang Y, Bian Y, Li Y, Liu L, Zhang H, Xie K, Wang G, Yu Y: Hydrogen Gas Protects Against Intestinal Injury in Wild Type But Not NRF2 Knockout Mice With Severe Sepsis by Regulating HO-1 and HMGB1 Release. Shock 2017, 48(3):364-370.

3.Taylor RW: Gut Motility Issues in Critical Illness. Crit Care Clin 2016, 32(2):191-201.

4.Klingensmith NJ, Coopersmith CM: The Gut as the Motor of Multiple Organ Dysfunction in Critical IIIness. Crit Care Clin 2016, 32(2):203-212.

5.Kelly DA: Intestinal failure-associated liver disease: what do we know today? Gastroenterology 2006, 130(2 Suppl 1):S70-77.

6.Reintam Blaser A, Malbrain ML, Starkopf J, Fruhwald S, Jakob SM, De Waele J, Braun JP, Poeze M, Spies C: Gastrointestinal function in intensive care patients: terminology, definitions and management. Recommendations of the ESICM Working Group on Abdominal Problems. Intensive Care Med 2012, 38(3):384-394.

7.Cicione C, Degirolamo C, Moschetta A: Emerging role of fibroblast growth factors 15/19 and 21 as metabolic integrators in the liver. Hepatology 2012, 56(6):2404-2411. 
8.Iwamoto J, Saito Y, Honda A, Miyazaki T, Ikegami T, Matsuzaki Y: Bile acid malabsorption deactivates pregnane X receptor in patients with Crohn's disease. Inflamm Bowel Dis 2013, 19(6):1278-1284.

9.Nolan JD, Johnston IM, Pattni SS, Dew T, Orchard TR, Walters JR: Diarrhea in Crohn's disease: investigating the role of the ileal hormone fibroblast growth factor 19. J Crohns Colitis 2015, 9(2):125131.

10.You M, Zhou Z, Daniels M, Jogasuria A: Endocrine Adiponectin-FGF15/19 Axis in Ethanol-Induced Inflammation and Alcoholic Liver Injury. Gene Expr 2018, 18(2):103-113.

11.Luo J, Ko B, Elliott M, Zhou M, Lindhout DA, Phung V, To C, Learned RM, Tian H, DePaoli AM et al: A nontumorigenic variant of FGF19 treats cholestatic liver diseases. Sci Transl Med 2014, 6(247):247ra100.

12.Goldstein B, Giroir B, Randolph A, International Consensus Conference on Pediatric S: International pediatric sepsis consensus conference: definitions for sepsis and organ dysfunction in pediatrics. Pediatr Crit Care Med 2005, 6(1):2-8.

13.Dellinger RP, Levy MM, Rhodes A, Annane D, Gerlach H, Opal SM, Sevransky JE, Sprung CL, Douglas IS, Jaeschke R et al: Surviving sepsis campaign: international guidelines for management of severe sepsis and septic shock: 2012. Crit Care Med 2013, 41(2):580-637.

14.Sutherland SM, Byrnes JJ, Kothari M, Longhurst CA, Dutta S, Garcia P, Goldstein SL: AKI in hospitalized children: comparing the pRIFLE, AKIN, and KDIGO definitions. Clin J Am Soc Nephrol 2015, 10(4):554-561.

15.Mutlu GM, Mutlu EA, Factor P: G/ complications in patients receiving mechanical ventilation. Chest 2001, 119(4):1222-1241.

16.Montejo JC: Enteral nutrition-related gastrointestinal complications in critically ill patients: a multicenter study. The Nutritional and Metabolic Working Group of the Spanish Society of Intensive Care Medicine and Coronary Units. Crit Care Med 1999, 27(8):1447-1453.

17.Reintam A, Parm P, Kitus R, Starkopf J, Kern H: Gastrointestinal failure score in critically ill patients: a prospective observational study. Crit Care 2008, 12(4):R90.

18.Lam SW, Nguyen NQ, Ching K, Chapman M, Fraser RJ, Holloway RH: Gastric feed intolerance is not increased in critically ill patients with type Il diabetes mellitus. Intensive Care Med 2007, 33(10):17401745.

19.Lebuffe G, Vallet B, Takala J, Hartstein G, Lamy M, Mythen M, Bakker J, Bennett D, Boyd O, Webb A: A european, multicenter, observational study to assess the value of gastric-to-end tidal PCO2 difference in predicting postoperative complications. Anesth Analg 2004, 99(1):166-172. 
20.Reintam Blaser A, Poeze M, Malbrain ML, Bjorck M, Oudemans-van Straaten HM, Starkopf J, GastroIntestinal Failure Trial G: Gastrointestinal symptoms during the first week of intensive care are associated with poor outcome: a prospective multicentre study. Intensive Care Med 2013, 39(5):899-909.

21.Gao Y, Yu KJ, Kang K, Liu HT, Zhang X, Huang R, Qu JD, Wang SC, Liu RJ, Liu YS et al: Procalcitionin as a diagnostic marker to distinguish upper and lower gastrointestinal perforation. World J Gastroenterol 2017, 23(24):4422-4427.

22.van Nieuwkoop C, Bonten TN, van't Wout JW, Kuijper EJ, Groeneveld GH, Becker MJ, Koster T, WattelLouis GH, Delfos NM, Ablij HC et al: Procalcitonin reflects bacteremia and bacterial load in urosepsis syndrome: a prospective observational study. Crit Care 2010, 14(6):R206.

23.Jiang L, Feng B, Gao D, Zhang Y: Plasma concentrations of copeptin, C-reactive protein and procalcitonin are positively correlated with APACHE II scores in patients with sepsis. J Int Med Res 2015, 43(2):188-195.

24.Liu D, Su LX, Guan W, Xiao K, Xie LX: Prognostic value of procalcitonin in pneumonia: A systematic review and meta-analysis. Respirology 2016, 21(2):280-288.

25.Jain S, Sinha S, Sharma SK, Samantaray JC, Aggrawal P, Vikram NK, Biswas A, Sood S, Goel M, Das M et al: Procalcitonin as a prognostic marker for sepsis: a prospective observational study. BMC Res Notes 2014, 7:458.

26.Coufal S, Kokesova A, Tlaskalova-Hogenova H, Snajdauf J, Rygl M, Kverka M: Urinary Intestinal Fatty Acid-Binding Protein Can Distinguish Necrotizing Enterocolitis from Sepsis in Early Stage of the Disease. J Immunol Res 2016, 2016:5727312.

\section{Tables}

Table 1. Baseline characteristics of patients with severe sepsis 


\begin{tabular}{|c|c|c|c|c|}
\hline & $\begin{array}{c}\text { Total } \\
(\mathrm{n}=90)\end{array}$ & $\begin{array}{l}\text { Sepsis } \\
(\mathrm{n}=58)\end{array}$ & $\begin{array}{l}\text { Sepsis-associated GI dysfunction } \\
\qquad(\mathrm{n}=32)\end{array}$ & $P$ \\
\hline Age, month & $19(5,60)$ & $17.5(5,57)$ & $19.5(6,68.5)$ & 0.739 \\
\hline Male, n (\%) & $51(56.7)$ & $31(53.4)$ & $20(62.5)$ & 0.407 \\
\hline PRISM III & $7(3,12)$ & $6(2,10)$ & $10(4,13)$ & 0.040 \\
\hline \multicolumn{5}{|l|}{ Complications } \\
\hline Shock, n (\%) & 42 & 19 & 23 & $<0.001$ \\
\hline Respiratory failure, n (\%) & 55 & 32 & 23 & 0.120 \\
\hline Liver dysfunction, $\mathrm{n}(\%)$ & 10 & 1 & 9 & $<0.001$ \\
\hline Acute kidney injury, n (\%) & 17 & 6 & 11 & 0.005 \\
\hline Infection site & & & & 0.011 \\
\hline Respiratory system, n (\%) & 44 & 33 & 11 & \\
\hline Gastrointestinal system, n (\%) & 21 & 7 & 14 & \\
\hline Urine system, n (\%) & 1 & 1 & 0 & \\
\hline Skin and soft tissue, , n (\%) & 11 & 9 & 2 & \\
\hline Central nervous system, n (\%) & 13 & 8 & 5 & \\
\hline Mechanical Ventilator, n (\%) & $53(58.9)$ & $31(53.4)$ & $22(68.8)$ & 0.158 \\
\hline Length of PICU stay, day & $13(7,20)$ & $12(7,16)$ & $16(11.5,35.5)$ & $<0.001$ \\
\hline PICU mortality, n (\%) & $11(12.2)$ & $5(8.6)$ & $6(18.8)$ & 0.160 \\
\hline
\end{tabular}

PRISM घ: pediatric risk of mortality $\square$ score; PICU: pediatric intensive care unit.

Table 2. The laboratory indexes of patients with sepsis.

\begin{tabular}{|c|c|c|c|c|}
\hline & $\begin{array}{c}\text { Total } \\
(\mathrm{n}=90)\end{array}$ & $\begin{array}{l}\text { Sepsis } \\
(\mathrm{n}=58)\end{array}$ & $\begin{array}{l}\text { Sepsis-associated GI dysfunction } \\
\qquad(\mathrm{n}=32)\end{array}$ & $P$ \\
\hline FGF19 $(\mu \mathrm{g} / \mathrm{ml})$ & $59.8(35.6,130.5)$ & $77.6(45.8,151.2)$ & $48.4(27.7,95.6)$ & 0.046 \\
\hline $\mathrm{WBC}\left(\times 10^{9} / \mathrm{L}\right)$ & $11.2(6,16.5)$ & $11.2(5.1,16.5)$ & $10.6(6.9,16.8)$ & 0.730 \\
\hline CRP (mg/L) & $16(5,78)$ & $8(5,70)$ & $25(6.5,93.5)$ & 0.123 \\
\hline PCT( ng/mL) & $1.0(0.1,7.9)$ & $0.5(0.1,5.7)$ & $4.5(0.6,12.6)$ & 0.005 \\
\hline $\mathrm{Lac}(\mathrm{mmol} / \mathrm{L})$ & $1.5(1,2.6)$ & $1.4(0.9,2.5)$ & $1.6(1.3,3.4)$ & 0.126 \\
\hline INR & $1.1(1.0,1.3)$ & $1.1(1.0,1.2)$ & $1.1(1.0,1.3)$ & 0.116 \\
\hline APTT(s) & $39(19.5,59.5)$ & $37(20,60.5)$ & $45.5(19,59.5)$ & 0.808 \\
\hline Fib (g/L) & $2.5(1.6,3.9)$ & $2.6(1.7,3.9)$ & $2.2(1.4,4.1)$ & 0.706 \\
\hline $\operatorname{ALT}(\mathrm{U} / \mathrm{L})$ & $21(14,43)$ & $21(15,35)$ & $20(12,63.5)$ & 0.701 \\
\hline TBIL $(\mu \mathrm{mol} / \mathrm{L})$ & $7.4(4.7,12.4)$ & $6.6(4.7,11.6)$ & $8.5(5.4,14.7)$ & 0.286 \\
\hline Albumin $(\mathrm{g} / \mathrm{L})$ & $38.5(32.5,41.2)$ & $39.6(34.5,43.1)$ & $36.4(28.3,40.3)$ & 0.025 \\
\hline $\mathrm{Cr}(\mu \mathrm{mol} / \mathrm{L})$ & $26(20,33)$ & $26(20,32)$ & $27(20.5,52.5)$ & 0.430 \\
\hline BUN (mmol/L) & $4(2.7,5.4)$ & $3.7(2.7,5.1)$ & $4.3(2.8,7.2)$ & 0.316 \\
\hline CK-MB (U/L) & $24(16,38)$ & $24(18,41)$ & $24(10,37)$ & 0.157 \\
\hline $\operatorname{PLT}\left(\times 10^{9} / \mathrm{L}\right)$ & $262.5(172,364)$ & $267(172,415)$ & $259(187.5,343.5)$ & 0.889 \\
\hline NK (\%) & $29.5(13.5,45.5)$ & $29(11,49)$ & $29.5(17,42.5)$ & 0.970 \\
\hline $\mathrm{CD}^{+} \mathrm{CD}^{+}(\%)$ & $1.6(1.0,2.0)$ & $1.5(0.8,1.9)$ & $1.7(1.3,2.1)$ & 0.267 \\
\hline CD19 (\%) $\square$ mean \pm sd & $35.1 \pm 13.5$ & $35.1 \pm 14.2$ & $35.1 \pm 12.5$ & 0.665 \\
\hline
\end{tabular}


FGF19: Fibroblast growth factor 19; WBC: white blood cell; CRP: C-reactive protein; PCT: procalcitonin; Lac: lactate; INR: international normalized ratio; APTT: activated partial thromboplastin time; Fib: fibrinogen; ALT:

alanine aminotransferase; TBIL: total bilirubin; Cr: creatinine; BUN: blood urea nitrogen; CK-MB: creatine kinase isoenzyme MB; PLT: platelet; NK: natural killer.

Table 3. Multivariate logistic regression about laboratory indexes with occurrence of GI disorder in patients with sepsis.

\begin{tabular}{lccccc}
\hline & \multicolumn{2}{c}{ Univariate logistic analysis } & & \multicolumn{2}{c}{ Multivariate logistic analysis } \\
\cline { 2 - 3 } \cline { 5 - 6 } & \multicolumn{1}{c}{$\boldsymbol{O R}(\mathbf{9 5 \%} \boldsymbol{C I})$} & $\boldsymbol{P}$ & & $\boldsymbol{O R}(\mathbf{9 5 \%} \boldsymbol{C I})$ & $\boldsymbol{P}$ \\
\hline FGF19 & $0.994(0.987,1.000)$ & 0.053 & & $0.992(0.984,0.999)$ & 0.046 \\
\hline PCT & $1.048(0.997,1.102)$ & 0.063 & & $1.042(0.988,1.099)$ & 0.126 \\
\hline Albumin & $0.919(0.856,0.985)$ & 0.018 & & $0.967(0.893,1.047)$ & 0.407 \\
\hline Lac & $1.216(0.944,1.566)$ & 0.129 & & $1.052(0.758,1.461)$ & 0.760 \\
\hline INR & $6.527(0.760,56.057)$ & 0.087 & & $4.914(0.211,114.606)$ & 0.306 \\
\hline PRISM III & $1.059(0.987,1.135)$ & 0.109 & & $1.064(0.979,1.157)$ & 0.125 \\
\hline
\end{tabular}

FGF19: Fibroblast growth factor 19; PCT: procalcitonin; Lac: lactate; INR: international normalized ratio; PRISM $\square$ : pediatric risk of mortality $\square$ score.

\section{Figures}




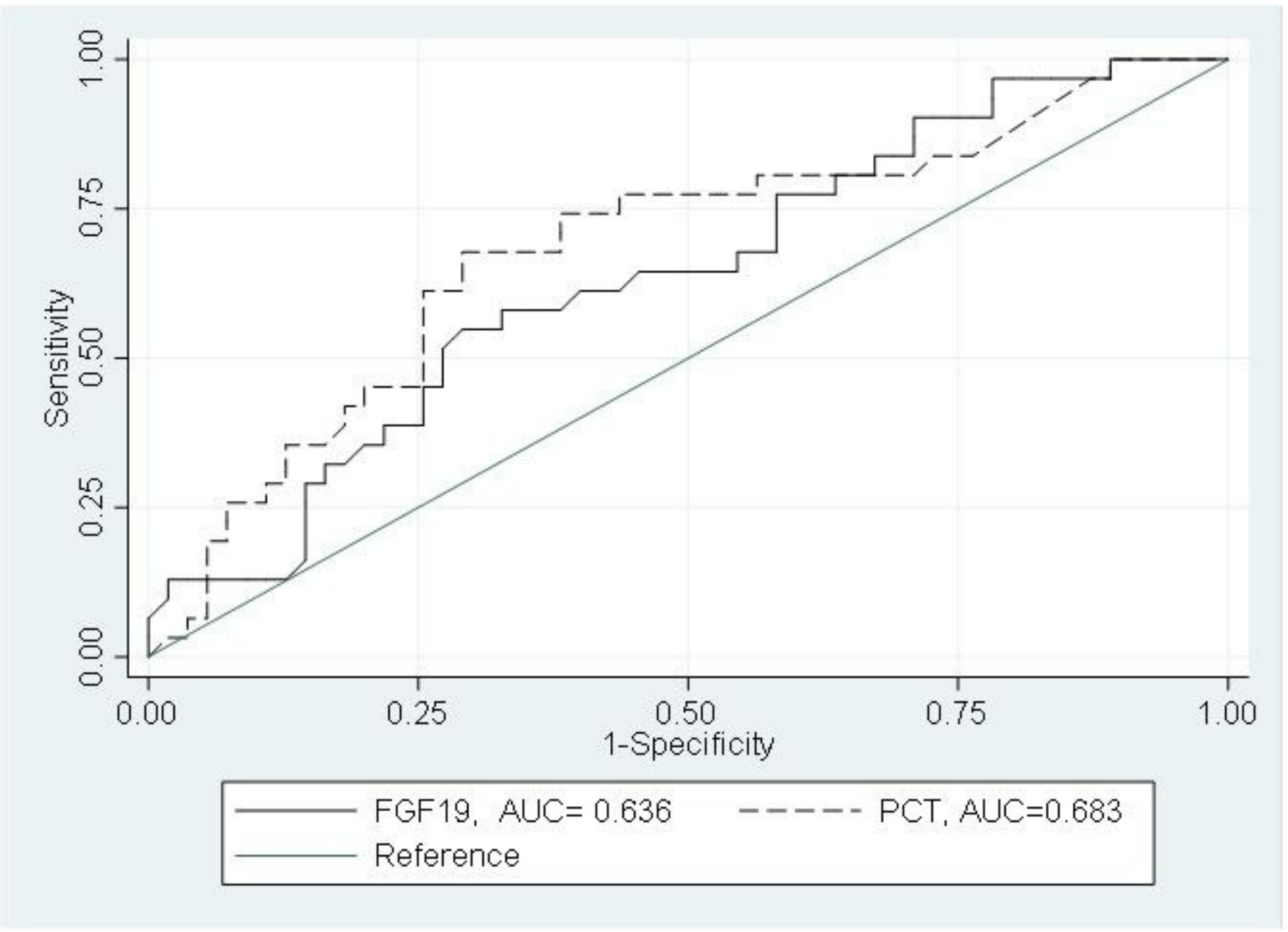

Figure 1

Receiver operating characteristic (ROC) analyses of serum fibroblast growth factor 19 (FGF19) and Procalcitonin (PCT) predicting gastrointestinal dysfunction in pediatric patients with sepsis. 
A

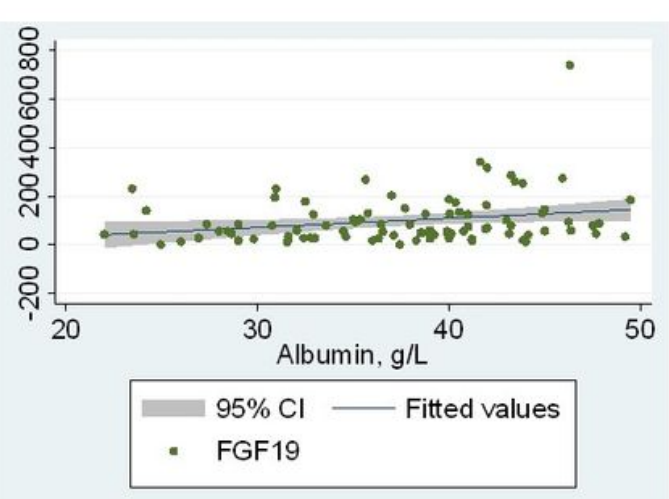

C

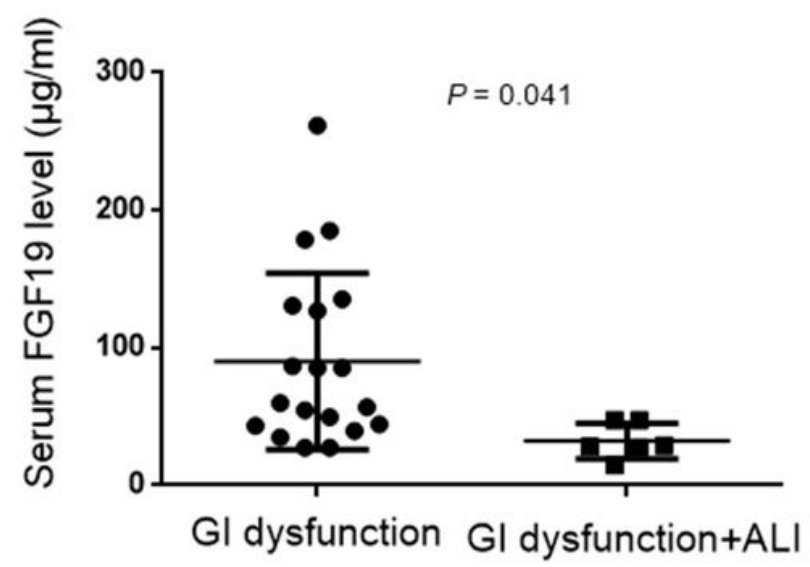

B

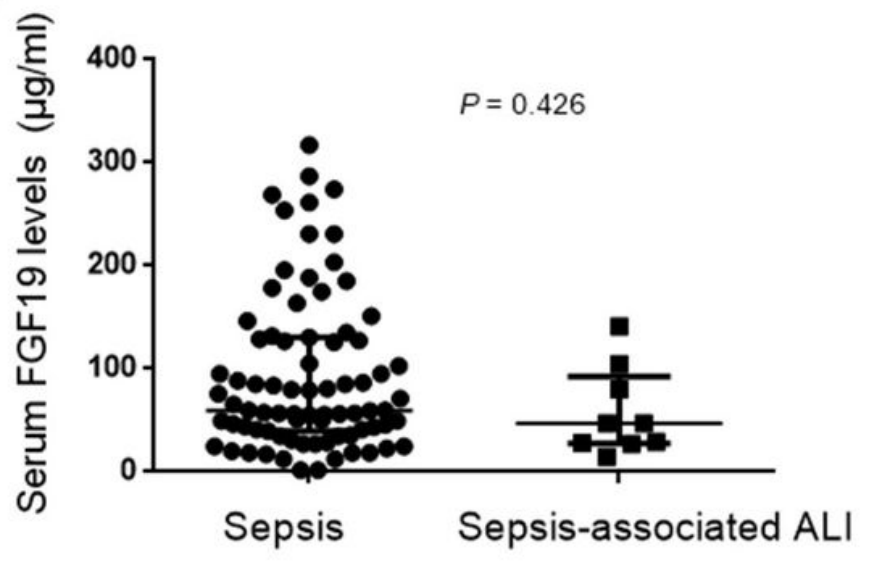

D

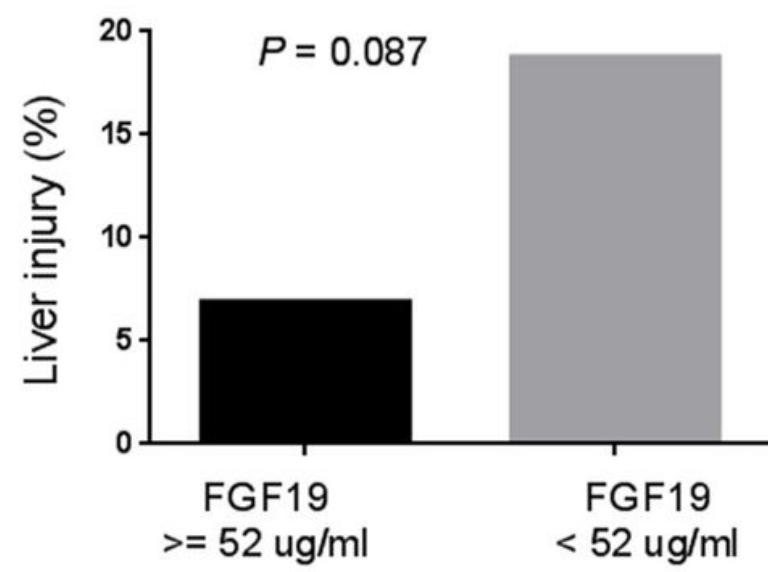

Figure 2

Levels of circulating fibroblast growth factor 19 (FGF19) are closely correlated to serum albumin levels and liver injury. (A) regression analysis between FGF19 and albumin, (B) serum FGF19 levels in total patients with or without liver injury, (C) serum FGF 19 levels in subgroup of patients with gastrointestinal (GI) dysfunction complicated by liver injury or not, (D) the rate of liver injury in different groups with FGF19 >= $52 \mu \mathrm{g} / \mathrm{mL}$ or FGF19 $<52 \mu \mathrm{g} / \mathrm{mL}$. 